\title{
Hybrid Organic-Inorganic photoresists, a promising class of materials for Optofluidic integration.
}

\author{
Jinane Elias ${ }^{1, *}$, Pascal Etienne ${ }^{1}$, Sylvie Calas-Etienne ${ }^{1}$ and Laurent Duffours ${ }^{2}$ \\ ${ }^{1}$ Laboratoire Charles Coulomb (L2C), University of Montpellier, CNRS, Montpellier, France \\ ${ }^{2}$ PRIME Verre, 1350 Albert Einstein, Montpellier, France
}

\begin{abstract}
Through the efforts to fuse planar optics and microfluidics in order to produce dye lasers, biosensors, trapping and cell sorting device, we can notice the rising interest in optofluidics since early and mid 2000's. However mass production of these devices heavily relies on fast and easy patterning of the constituent material. PDMS, being one of these materials, gained an added value because of its elasticity, hydrophobicity and permeability to gaz. Nonetheless, these specifications are not convenient for all types of applications. The growing capability to use Hybrid Organic-Inorganic materials for the fabrication of integrated optics components and microfluidic channels is what makes this class of materials an ideal candidate for this integration. This work aims to implement, on the same chip, an optical and a microfluidic layer using Sol-Gel processing of Organic-Inorganic materials. The interest in this vertical integration arises from the need to manipulate the fluid in the microchannels using evanescent field optical pressure.
\end{abstract}

\section{Introduction}

Optofluidics refer to a wide range of applications in which optics and fluidics are integrated on the same device and used synergistically to synthesize creative, novel and highly versatile functionalities, in which light is used to manipulate and analyse fluids or vice versa. Even though the integration of an optical component (light sources, filters or photodetectors) into the lab-ona-chip has recently gained lots of interest, with the efforts to fuse planar optics and microfluidics, their commercialization remains greatly challenged by the ability to mass produce these devices while maintaining low cost hence disposability.

PDMS appeared to be the best candidate for mass production of these devices. However it presents some drawbacks, such as absorption of organic solvents, lipidic like and hydrophobic molecules and subsequent swelling of the chip, permeability to gas, and minor auto-fluorescence (in comparison with other polymers) in the Near UV-Vis-Near IR $(240-1100 \mathrm{~nm})$. These drawbacks limit its use to certain applications where these problems don't affect the final output. These problems, especially the hydrophobic nature of PDMS, have triggered many research projects in order to modify the surface of PDMS using special coatings. As an alternative, E. Mechref et al. have developed an organicinorganic positive photoresist [1], suitable for microfluidic channels fabrication using single step (deposition, insulation, and engraving) sol-gel processing. This material can be tailored to be as good as PDMS and can still overcome its undesired properties. One problem arises, which is the control of fluids in these rigid micro channels and the implementation of the basic microfluidic components such as active mixers, valves and sensors. What we propose as a solution is evanescent field optical control through an integrated optical layer which was already implemented in previous works in our Lab[2] Our main purpose is to design and implement optofluidic control elements for lab on chip applications such as optofluidic mixer as a first step.

\subsection{Hybrid Organic Inorganic Materials}

\subsubsection{Hybrid materials definition}

As the name suggests they are a set of material in which two networks coexist: an organic and inorganic one, giving rise to a hybrid material that can be tailored, in a way to manifest the properties of both its constituents. The inorganic component increases hardness and resistance to elevated temperature while the organic network ensures good elasticity, tenacity, ductility which allows us to increase the thickness of the coatings without significant cracks. Precursors are ORganically MOdified CERamics having a general chemical formula of $R_{x} M(O R)_{4-x}$. The alkoxide group OR' is responsible of the mineral network elaborated by sol-gel process: a sol that turns into a gel after hydrolysis and condensation reactions. The $\mathrm{R}$ groups are responsible for the organic network elaborated through selective UV induced polymerisation or through thermally induced polymerization.Due to their unique and intermediate properties of the mineral and organic, these materials are of great interest for many applications in optics, and microfluidics.

\subsubsection{Negative Tone Hybrid photoresists for integrated optics}

Since 1992 the work of different groups has shown the effectiveness of a composition based on a mixture of methacryloxypropyltrimethoxysilane (MAPTMS) and zirconium propoxide $\left(\mathrm{Zr}(\mathrm{OPr})_{4}\right.$. The addition of $\mathrm{Zr}(\mathrm{OPr})_{4}$ to the PMMA network is used to adjust its optical properties, notably to increase the refractive index. It also enhances the quality of the waveguide profile after 
revelation because it leads to a well-polymerised and hydrophilic surface under UV-illumination. Subsequent studies have shown the potential of this system to yield an index contrast of 0.013 [2] with the addition of an adequate photoinitiator. It was shown that the higher the refractive index contrast, the more intense the evanescent field can be [3]. For this reason we chose to proceed with a mixture of MAPTMS and $\mathrm{Zr}(\mathrm{OPr})_{4}$ while trying to increase the contrast by changing the photoinitiator and the $\mathrm{Zr}(\mathrm{OPr})_{4}$ to MAPTMS proportion. In addition, this simple fabrication process of waveguides is very adequate to mass production.

\subsubsection{Positive Tone Hybrid photoresists for microfluidic chips}

A positive tone resist is a good alternative for negative tone photoresists when it comes to the processing of channels by laser writing. A hybrid material offers better command on the properties of the microfluidic channels because of the reasons cited in 1.1.1. This is why E. Mechref et al. [1] developed an organic-inorganic chemically amplified positive photoresist based on Polyamic acid (PAA) and suitable for microfluidic channels fabrication using single step (deposition, insulation, and engraving) sol-gel process. PAA is well known for its good mechanical properties and high thermal stability, enhanced even more through the addition of 4-vinylether-phenyltriethoxysilane (VEPTES) pre-hydrolyzed by sol-gel method to form the inorganic network.

\subsection{Evanescent Field Optical Control}

Light carries energy and momentum; optical forces result from the transmission of this energy to another system. On the macroscopic scale, these forces pass unnoticed. However, the acceleration induced by a light beam with a moderate intensity, on relatively small particles is effective to move, trap, or guide a particle in the Mie or the Rayleigh regime. This became experimentally feasible after the invention of lasers and has been subject to implementation since early 1970's in the work of Ashkin [4]. Kawata and Sugiura [5]reported that particles with diameters of $1-27 \mu \mathrm{m}$ present within the penetration depth of the evanescent field can be attracted to the core by the gradient force and propelled along a single mode fiber by the scattering force in the same direction as the mode propagates. Theoretical examination of optical force exerted on a dielectric sphere by the Gaussian beam the evanescent field was also conducted.

\section{Results}

Initial studies have demonstrated the feasibility of single mode waveguides written due to selective photo-induced increase of refractive index in a hybrid organic-inorganic negative type photo resist, composed of hydrolysed MAPTMS and $\mathrm{ZrPO}_{4}$ chelated by MAAH and further hydrolysed. After UV curing it showed an index rise of around 0.002 in the visible. Refractive index measurements were made using an ATAGO's MultiWavelength Abbe Refractometer DR-M2 having an instrumental error of $\pm 5 \times 10^{-5}$. Unfortunately the statistical error appears to be of the same order of the difference which makes the significance of these results doubtable. Another measurement method is under investigation. Light confinement in these waveguides was confirmed. Attenuation in the visible range (at $625 \mathrm{~nm}$ ) will be quantified, alongside with the attenuation due to bending. Other mixtures such as (3 Glycidyloxypropyl)trimethoxysilane (GPTMS) were investigated; it showed on the other hand a decrease in the refractive index due to insulation.

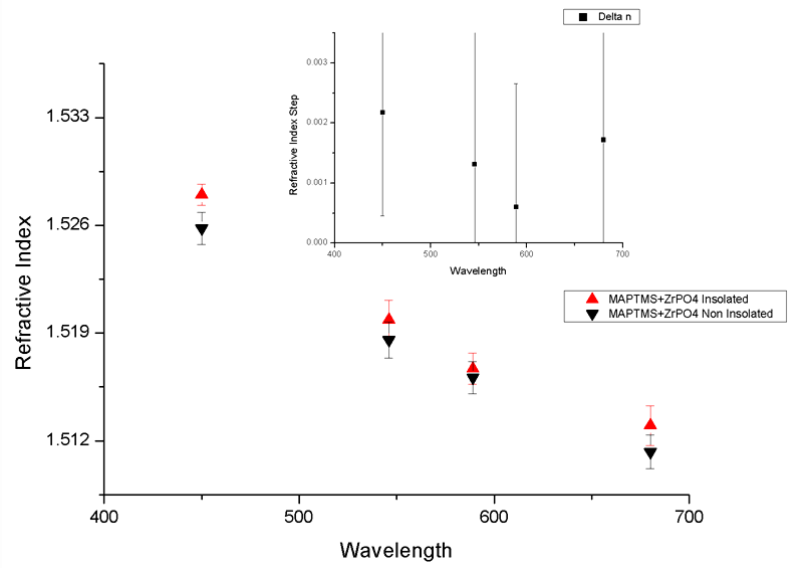

Figure 1: Refractive Index of cured and non cured MAPTMS (annealed at 110 degrees Celsius) and refractive index step (Inset)

\section{Conclusion}

As a conclusion, hybrid organic-inorganic materials are biocompatible, mechanically robust and impermeable materials suitable for mass production of optofluidic Lab on Chips. Waveguides can be easily written by photopolymerisation of negative photoresists, and microfluidic channels revealed in positive resist after laser writing. All of which makes it a low cost, high yield process.

\section{References}

[1] E. Mechref, J. Jabbour, S. Calas-Etienne, K. Amro, A.Mehdi, R. Tauk, D. Zaouk , P. Etienne, RSC Adv., 6 (2016)

[2] P. Coudray, P. Etienne, Y. Moreau, J. Porque, S. I. Najafi, Opt. Commun., 143 (1997)

[3] R. Bernini, N. Cennamo, A. Minardo, and L. Zeni, , IEEE Sens. J.,6 (2006)

[4] A. Ashkin, Phys. Rev. Lett., 24 (1970)

[5] S. Kawata and T. Sugiura, Opt. Lett., 17 (1992) 